\title{
Guidelines for tracheostomy in COVID-19 pandemic
}

\author{
Meenesh Juvekar ${ }^{1 *}$, Baisali Sarkar ${ }^{2}$ \\ Bombay Hospital Medical and Research Centre and Grant Medical College and JJ Group of Hospitals, Mumbai, \\ Maharashtra, India
}

Received: 29 September 2020

Accepted: 05 November 2020

\section{*Correspondence:}

Dr. Meenesh Juvekar,

E-mail: meeneshj@gmail.com

Copyright: ( ) the author(s), publisher and licensee Medip Academy. This is an open-access article distributed under the terms of the Creative Commons Attribution Non-Commercial License, which permits unrestricted non-commercial use, distribution, and reproduction in any medium, provided the original work is properly cited.

\begin{abstract}
Measures to be taken by the clinicians involved in doing tracheostomy in light of the recent coronavirus disease, 2019 pandemic are highlighted in current article. Current findings about coronavirus disease, 2019 are evaluated and guidelines to be taken while performing tracheostomy are reviewed. If guidelines for tracheostomy are strictly followed, the probability to get COVID-19 infection drastically reduces. COVID-19 disease has now become pandemic. Many patients admitted in the intensive care require tracheostomy. The aerosol spread of this disease greatly increases the spread and risk of amongst medical and healthcare staff. The protocols for tracheostomy should be clearly defined and followed prior to doing the procedure. This review summarizes the protocols for tracheostomy required to prevent the spread of the disease.
\end{abstract}

Keywords: SARS-CoV-2, Covid-19, Pandemic, Tracheostomy, Guidelines

\section{INTRODUCTION}

SARS-CoV-2 (severe acute respiratory syndrome coronavirus 2) is the causative agent for coronavirus disease 2019. ${ }^{1}$ Coronavirus causes respiratory tract infections. The severity can be mild like common cold, or can be lethal, like SARS, MERS (middle east respiratory syndrome) and COVID-19 (corona virus disease, 2019). COVID-19 disease was first identified in December 2019 in Wuhan, the capital of China's Hubei province and it caused a pandemic. ${ }^{2,3}$ The virus is most contagious when people are symptomatic, although spread is possible even before symptoms appear. ${ }^{4}$ The virus mainly spreads by close contact via small droplets produced while coughing, sneezing and talking.,

Aerosol transmission occurs while doing intubation and cardiopulmonary resuscitation. Fomite transmission is also seen. ${ }^{4}$ The incubation period is 5 to 6 days but may range from 2 to 14 days. The virus survives for hours to days on surfaces. The patient may be asymptomatic or present with flu like symptoms like fever, cough, sneezing, fatigue and shortness of breath. The disease may progress to pneumonia, multi-organ failure, and even death..$^{8-11}$ Olfactory dysfunction of sudden onset is the most common early clinical feature of COVID-19. ${ }^{12,13}$

Many patients require intensive care and subsequent intubation with tracheostomy. This procedure can greatly increase the aerosol spread to the healthcare staff and surrounding patients. Hence, appropriate protective and hygiene measures are of utmost improtant. ${ }^{6,7}$

\section{METHODS}

\section{Data sources}

Protocols and guidelines relating to tracheostomy in this COVID-19 pandemic were identified from webpages of otolaryngology societies such as British laryngological association, centers for disease control and prevention 
(CDC), web of science, pubMed, Google scholar and various other literatures.

\section{Protocol}

The current situation of COVID-19 pandemic has lead to increase in critically ill patients needing hospitalization and ICU care. Many cases require prolonged ventilatory support, urging the need for tracheostomy. Tracheostomy is an aerosol generating procedure, with potential risk to the doctors and healthcare staff to get infected by SARSCoV-2 virus during the operation and post-operative care of the tracheostomy site. Thus, selection of patient for doing tracheostomy, timing of tracheostomy, performing tracheostomy and after care is very important in this current COVID-19 scenario.

\section{Decisions for tracheostomy}

Amongst all the patients getting hospitalized, 8 to $13 \%$ patients are critically ill and require mechanical ventilation and tracheostomy for life support. ${ }^{14}$ The indications for tracheostomy are mainly assistance of mechanical ventilation for a long period, simultaneously reducing complications due to endotracheal tube and fasten the weaning from ventilation. Decision for tracheostomy should be made in the mentioned protocol; two ICU consultants should make a decision after discussion with the surgical team and senior anesthetist. The patient should have a good expectation of achieving complete recovery and having an independent lifestyle. Tracheostomy is indicated in laryngeal injury, trauma or dysfunction, ventilator associated pneumonia, ventilator associated respiratory muscle atrophy, cumulative effect of sedation etc. Tracheostomy is not recommended in patients who still need high ventilator requirements, high fractions of inspired oxygen ( $\mathrm{FiO} 2)$, multiorgan failure and who might require prone positioning. ${ }^{15}$

\section{Timing for tracheostomy}

Delaying tracheostomy in a COVID-19 patient in order to reduce risks to the health-care staff results in prolongation in the duration of sedation and intubation, which in turn can result in additional complications. Tracheostomy should be delayed for at least 10 days of mechanical ventilation and should be considered only when patients are showing signs of clinical improvement. The patient should be relatively stable and should tolerate laying flat with periods of brief apnea. In ICU all the critically ill patients who need tracheostomy should undergo preoxygenation followed by a trial of apnea with a $\mathrm{FiO} 2$ of 1.0 and positive end expiratory pressure of 5 $\mathrm{cm} \mathrm{H} 2 \mathrm{O}$. Rapid desaturation, indicates a high risk factor and tracheostomy should be deferred in such cases. ${ }^{16}$

\section{Place for tracheostomy}

Whenever possible tracheostomy should be performed in the operation theatre. The operating room should have negative pressure environment with high frequency air changes (25 per hour). Each operating room should have separate ventilating system with integrated high efficiency particulate air (HEPA) filter. Separate operating room should be designated for COVID-19 suspected or positive patients. There should be negative pressure isolation transfer cabin to transfer patients between the isolation ward and operation theatre. Those who are involved must wear level 3 protective medical equipment (BSL-3). Principles of clean area, contaminated pollution area and two buffer zones should be followed while entry and exit into the operating theatre. Alternatively, a provisional operating room with negative pressure can be set up in the ICU. If negative pressure theatre is not available then consider a normal theatre with closed doors during the procedure. Consider turning off laminar flow (if present). ${ }^{17}$

\section{Staff preparation}

All staff must wear personal protective equipment (PPE) like cap, powered air purifying respirator (PAPR), eye protection, face shield, fluid-repellent disposable surgical gown, double gloves, shoe cover. If PAPR is not available then FFP3 mask (fit-tested filtering face piece 3) or N95 mask is used with an additional fluid shield. A core COVID airway team is formed to facilitate efficiency during the crisis. Suggested COVID-19 airway team included ENT/head \& neck consultant, senior ICU anesthesia consultant, registrar, senior theatre nurse. ${ }^{18-22}$

\section{Equipment for tracheostomy}

Pre-prepared tracheostomy sets are kept in grab bags and stored in a designated clean zone. The equipment includes tracheostomy set, cuffed non-fenestrated tracheostomy tubes of appropriate sizes and HME (simple heat and moisture exchange filter) with viral filter. Only use cuffed, non-fenestrated tracheostomy tubes. Ensure that there is adequate supply of cuffed, non-fenestrated tracheostomy tubes of various sizes. Establish a system to record what has been used and what needs restocking. Attach syringe to tracheostomy balloon ready for inflation. Consider preloading the HME onto the inner tube. Ensure only closed in-line suction is used for ETT (endotracheal tube) and tracheostomy tube. ${ }^{23}$

\section{Tracheostomy procedure}

Approach to tracheostomy can be surgical, percutaneous or hybrid. Percutaneous tracheostomy involves opening of the ventilator circuit more commonly than the surgical tracheostomy and it is done with the help of ultrasound and bronchoscopy guidance, thus improving safety to the patient but it generates more aerosol as compared to the surgical approaches. Hence, surgical tracheostomy approach is more favored over percutaneous tracheostomy approach during COVID-19 pandemic. ${ }^{24,25}$ Single-use bronchoscopes along with a sealed ventilator circuits are preferably used while doing percutaneous tracheostomies. 


\section{Positioning of patient while doing tracheostomy}

The patient lies in supine position with hyper-extended head and face and is covered by drapes. Consider use of surgical ties rather than diathermy, to prevent vapour fumes containing viral particles. Use of smoke evacuator is recommended while using open approach tracheostomy. ${ }^{28}$

\section{Before opening of trachea}

Inform anesthetist of readiness to open trachea. Confirm paralysis and request full paralysis throughout to reduce risk of cough. Neuromuscular blocking drugs are used to paralyze the muscles thus eliminating undue coughing (this reduces the aerosol spread) and movement of patient during the procedure. However it should be kept in mind that critically ill patients may land up in tachyphylaxis in response to neuromuscular blocking drugs. ${ }^{26,27}$ Preoxygenation with PEEP (positive end expiratory pressure) is done then ventilation stopped. This allows time for passive expiration with open APL (adjustable pressure limiting) valve. Consider clamping of the ETT then advance cuff beyond proposed tracheal window. Hyperinflate cuff and re-establish oxygenation with PEEP. Adequate oxygenation should be done prior to opening of the trachea, reducing the duration of apnoea. ${ }^{28}$

\section{Creating tracheal window}

One must create the tracheal window as much cranially as possible (e.g. $1^{\text {st }}$ or $2^{\text {nd }}$ inter-tracheal space) taking care to avoid the ETT cuff injury. Turn off flow with open APL valve, allowing passive expiration, after clamping ETT. Deflate ETT cuff and withdraw back proximally to the tracheal window under direct vision. Ensure tracheal window is of sufficient size to allow easy insertion of tracheostomy tube without injury to cuff. The anterior tracheal wall can be sutured to the skin to facilitate insertion and post-operative management. Insert cuffed, non-fenestrated tracheal tube through the tracheal window. Immediately inflate tracheostomy tube cuff. Replace introducer with non-fenestrated inner tube and HME filter. Prompt attachment of circuit is done. Resume ventilation immediately. ${ }^{28}$

\section{Confirmation of tracheostomy tube}

Confirm position of the tube while patient lying in a 30 degree head up (ICU nursing) position. Confirm position of tracheostomy tube with end-tidal $\mathrm{CO} 2$ only, thus avoiding contamination of stethoscope by auscultation. Withdraw clamped ETT carefully. Secure tube with sutures and tapes.

\section{Proper disposal of equipment and decontamination of operation theatre}

After tracheostomy is completed, disposal of PPE kit needs to be done according to local guidelines. After completion of tracheostomy, the full anesthesia unit should be disinfected for two hours with $12 \%$ hydrogen peroxide present inside an anesthesia circuit sterilizer. Chlorine containing disinfectant $(2,000 \mathrm{mg} / \mathrm{L})$ is used to clean the floor of operation theatre and all the reusable medical equipments. Surgical instruments are soaked in $2,000 \mathrm{mg} / \mathrm{l}$ chlorine-containing disinfectant and then sealed in double-layered disposable waste bags before being sent to the respective disinfection area. The air purification system of operation theatre is shut down after 30 minutes and then an ultra-low volume 20-30 $\mathrm{mL} /$ minute of $3 \%$ hydrogen peroxide is used to fumigate the OT for two hours, after which the negative pressure ventilation is turned on again. ${ }^{29}$

\section{Post-operative care}

There is high risk of aerosol transmission after tracheostomy. Thus patient care should be balanced along with the safety of health-care staff. Patients need to be nursed and supervised by experienced staff who are well trained in post-operative tracheostomy care. ${ }^{30}$ Tracheostomy tube care is done in BSL-3 settings. Patients should be transferred cautiously. One dedicated team member is allocated for holding the tracheostomy tube whenever the patient is being prone or turned. Humidified oxygen is avoided if possible, instead HME (simple heat and moisture exchange filter) are used. ${ }^{31}$ Use only in line closed suction circuits at all times. Cuff pressures are maintained at $20-30 \mathrm{~cm}$ of $\mathrm{H} 2 \mathrm{O}$ and checked every 12 hours. ${ }^{32}$ Cuff should not be deflated, considering risks to patient, staff and the environment. Dressings should not be changed unless there is a frank sign of infection or bleeding. First tube change is delayed up-to seven to ten days. While changing the tube, all should wear full PPE kits and perform same sequence with pause in ventilation before deflating cuff and inserting new tube with immediate re-inflation of the cuff. Cuffed non-fenestrated tube should be used until the patient is confirmed COVID-19 negative. Subsequent planned tube changing should be at 30 days interval. If patient is confirmed COVID-19 negative, then he is moved to a COVID-19 negative ward and then considered trials of cuff deflation. Increasing cuff deflation period, promotion of coughing, gradual vocalization and rehabilitation of swallowing is done before weaning the patient off the tracheostomy. However, all of these have a potential to spread aerosol. ${ }^{28}$ To prevent the spread of aerosol, face masks and tracheostomy shields are used. ${ }^{33}$ Planning for decannulation should be made in co-ordination with physiotherapists. Before decannulation, the patient has to tolerate 24 hours with cuff deflated and 12 hours with one way valve, with minimal or no tracheal suctioning requirements. He should be able to independently cough out secretions without any evidence of any bronchopulmonary infection, upper airway obstruction or high oxygen requirements. ${ }^{34}$ Patients with suspected hyper-inflammatory conditions and lung inflammation signs should be considered for immunomodulation therapy with methylprednisolone. ${ }^{35}$ 


\section{RESULTS}

Prasun et al from India reported tracheostomies in 11 COVID-19 positive patients, of which 8 were male and 3 were female. 9 patients were more than 50 years of age. All the patients had one or more comorbidities in the form of diabetes mellitus, renal disease and cardiac problems. The indications for tracheostomy in all patients were pneumonia needing prolonged intubation. Tracheostomy was done 10 days after prolonged mechanical ventilation. All patients tolerated the procedure well. ${ }^{37}$

Chao et al studied 53 COVID-19 patients who underwent tracheostomy, the average time from endotracheal intubation to tracheostomy was 19.7 days \pm 6.9 days. The most common indication for tracheostomy was acute respiratory distress syndrome, followed by failure to wean ventilation and post-extracorporeal membrane oxygenation decannulation. Both open surgical and percutaneous tracheostomy techniques were performed utilizing methods to mitigate aerosols. Importantly no healthcare worker transmissions were reported by those involved in the procedure. ${ }^{38}$

New York university thorasic group did a study on 270 COVID-19 patients who required invasive mechanical ventilation. Of those, 98 patients underwent percutaneous dilational tracheostomy. The mean time from intubation to the procedure was $10.6 \pm 5$ days. During this period none of health care providers had symptoms or tested positive for COVID-19. ${ }^{39}$

Thus, it is suggested to do tracheostomy after 10 days of intubation. Special guidelines and steps of tracheostomy reduce the spread of aerosol to a large extent. Postoperative tracheostomy care should be done by experienced staff. The cuff of tracheostomy tube should remain inflated unless indicated. Change of tube is preferably done after 10 days. Decannulation is done only when the patient becomes COVID-19 negative. The above is especially important in the Indian scenario where there are a very high number of patients with comorbidities.

\section{DISCUSSION}

Wang et al from China did a study on 344 COVID-19 patients in the ICU. Of the 344 subjects, 133 patients expired on the $28^{\text {th }}$ day with a median survival of 25 days. Of these patients, there were many with different comorbidities with 141 patients having hypertension. In survivors, the mean duration of hospitalization for a negative test result was 12 days. ${ }^{40}$ Zhang et al conducted another study in China on 633 COVID-19 patients, of whom 247 patients had at least one comorbidity and concluded that older individuals, greater than 60 years, were more likely to have a complicated prognosis. ${ }^{41}$ Similarly Garg et al from United States used COVIDNET in 14 states to monitor the demographics of
COVID-19 patients who were hospitalized. From March $1^{\text {st }}$ to $30^{\text {th }} 2020$, there were a total of 180 patients on COVID-NET of which $89.3 \%$ of the patients had an underlying comorbidity. The most common comorbidities found were obesity, hypertension and diabetes mellitus. ${ }^{42}$ Thus patients with COVID-19 disease who have comorbidities such as hypertension or diabetes mellitus are more likely to develop a more severe course and progression of the disease. Furthermore, elderly patients, especially those above 65 years with comorbidities may require prolonged ventilation and tracheostomy. COVID19 disease has spread worldwide with ENT doctors at high risk of getting infected. The aerosol spread can not only occur during tracheostomy procedure but also while maintaining the tracheostomy site. Hence the infective duration of the patient must be noted and proper precautions and guidelines should be followed to reduce the risk of spread.

\section{CONCLUSION}

COVID-19 disease is highly contagious disease. Measures need to be taken to prevent its transmission thus attaining a plateau and decline in the disease spread. This review summarizes the protocols for tracheostomy in the current scenario. As many patients may require tracheostomy, a carefully planned approach is required keeping the protocols in mind, choosing the right patient and evaluating the risks involved.

\section{Funding: No funding sources \\ Conflict of interest: None declared \\ Ethical approval: Not required}

\section{REFERENCES}

1. Coronavirus disease 201, symptoms and causes mayo Clinic. Available at: https://www.mayoclinic. org/diseases-conditions/coronavirus/symptomscauses/syc-20479963. Accessed on 14 April 2020.

2. David SH, Esam IA, Tariq AM, Francine N, Richard K, Osman D, et al. The continuing 2019-nCoV epidemic threat of novel coronaviruses to global health- The latest 2019 novel coronavirus outbreak in Wuhan, China. Int J Infect Dis. 91:264-66.

3. Director-General's opening remarks at the media briefing on COVID-19, World Health Organization. Available at: https://www.who.int/dg/speeches/ detail/who-director-general-s-opening-remarks-atthe-media-briefing-on-covid-19-11-march-2020. Accessed on 12 March 2020.

4. How COVID-19 Spreads centers for disease control and prevention. Available at: https://www.cdc.gov/ coronavirus/2019-ncov/prevent-getting-sick/howcovid-spreads.html. Accessed on 3 April 2020.

5. European centre for disease prevention and control. Available at: https://www.ecdc.europa.eu/en/covid19/questions-answers. Accessed on 30 April 2020. 
6. Anagiotos A, Petrikkos G. Otolaryngology in the COVID-19 pandemic era: the impact on our clinical practice. Eur Arch Otorhinolaryngol. 2020;1-8.

7. Doremalen N, Bushmaker T, Morris DH, Holbrook MG, Gamble A, Williamson BN, et al. Aerosol and Surface Stability of SARS-CoV-2 as Compared with SARS-CoV-1. N Engl J Med. 2020;382(16):1564-7.

8. Nanshan C, Min Z, Xuan D, Jieming Q, Fengyun G, Yang $\mathrm{H}$, et al. Epidemiological and clinical characteristics of 99 cases of 2019 novel coronavirus pneumonia in Wuhan, China: a descriptive study. Lancet. 2020;395(10223):507-13.

9. Symptoms of coronavirus centers for disease control and prevention. Available at: https://www.cdc.gov/ coronavirus/2019-ncov/symptoms-testing/ symptoms.html. Accessed on 18 June 2020.

10. Novel coronavirus information center: expert guidance and commentary. Available at: https://www.elsevier.com/connect/coronavirusinformation-center. Accessed on 18 June 2020.

11. Grant MC, Geoghegan L, Arbyn M, Mohammed Z, McGuinness L, Clarke EL, et al. The prevalence of symptoms in 24,410 adults infected by the novel coronavirus (SARS-CoV-2; COVID-19): A systematic review and meta-analysis of 148 studies from 9 countries. PLoS One. 2020;15(6):e0234765.

12. Lechien JR, Chiesa-Estomba CM, De Siati DR, Horoi M, Le Bon SD, Rodriguez A, et al. Olfactory and gustatory dysfunctions as a clinical presentation of mild-to-moderate forms of the coronavirus disease (COVID-19): a multicenter European study. Eur Arch Otorhinolaryngol. 2020;277(8):2251-61.

13. Heidari F, Karimi E, Firouzifar M, Khamushian P, Ansari R, Mohammadi Ardehali M, Heidari F. Anosmia as a prominent symptom of COVID-19 infection. Rhinology. 2020;58(3):302-3.

14. Mehta AB, Syeda SN, Bajpayee L, Cooke CR, Walkey AJ, Wiener RS. Trends in tracheostomy for mechanically ventilated patients in the United States. Am J Resp Crit Care Med. 2015;192:446-54.

15. Mamary AJ, Kondapaneni S, Vance GB, Gaughan JP, Martin UJ, Criner GJ. Survival in Patients Receiving Prolonged Ventilation: Factors that Influence Outcome. Clin Med Insights Circ Respir Pulm Med. 2011;5:17-26.

16. Mishra P, Jedge P, Kaushik M, Artham P, Kumari S. Our Experience of Tracheostomy in COVID-19 Patients . Indian J Otolaryngol Head Neck Surg. 2020;1-4.

17. Wong J, Goh QY, Tan Z, Lie SA, Tay YC, Ng SY, et al. Preparing for a COVID-19 pandemic: a review of operating room outbreak response measures in a large tertiary hospital in Singapore. Se préparer pour la pandémie de COVID-19: revue des moyens déployés dans un bloc opératoire d'un grand hôpital tertiaire au Singapour. Can J Anaesth. 2020;67(6): 732-45.

18. Guidance for ENT surgeons during the COVID-19 pandemic. Available at: https://www.ahns.info/wpcontent/uploads/2020/03/Australian-Guidance-for-
ENT-surgeons-during-the-COVID.pdf. Accessed on 20 March 2020.

19. General guidance for clinicians in Ireland dealing with head and neck cancer during the current COVID-19 outbreak Irish head and neck society. Available at: https://www.ahns.info/wpcontent/uploads/2020/03/Irish-Head-and-NeckSociety-considerations-on-COVID-20-3-20.pdf. Accessed on 10 April 2020.

20. COVID-19: considerations for optimum surgeon protection before, during, and after operation College of Surgeons. Available at: https://www.facs.org/ covid-19/clinical-guidance/surgeon-protection. Accessed on 10 April 2020.

21. Lu D, Wang H, Yu R, Yang H, Zhao Y. Integrated infection control strategy to minimize nosocomial infection of coronavirus disease 2019 among ENT healthcare workers. J Hosp Infect. 2020;104(4):4545 .

22. Synopsis of the Toronto approach. Available at: https://www.ahns.info/wp-content/uploads/2020/ 03/Jonathan-Irish-Synopsis-of-the-TorontoApproach.pdf. Accessed on 10 April 2020.

23. Preventing infections in patients with tracheostomy. Available at: https://tracheostomyeducation.com/ infection-control-issues-in-caring-for-patients-withtracheostomy. Accessed on 10 April 2020.

24. Tien HC, Chughtai T, Jogeklar A, Cooper AB, Brenneman F. Elective and emergency surgery in patients with severe acute respiratory syndrome. Can J Surg. 2005;48:71-4.

25. Chee VW, Khoo ML, Lee SF, Lai YC, Chin NM. Infection control measures for operative procedures in severe acute respiratory syndrome-related patients. Anesthesiol. 2005; 100:1394-8.

26. Haddad S. Tachyphylaxis to cisatracurium- case reports and literature review. Middle East $\mathbf{J}$ Anaesthesiol. 2008;19:1079-92.

27. Tschida SJ, Graupe KJ, Hoey LL, Vance-Bryan K. Resistance to nondepolarizing neuromuscular blocking agents. Pharmacotherapy. 1996;16:409-18.

28. Brendan AM, Brenner MJ, Warrillow SJ. Tracheostomy in the COVID-19 era: global and multidisciplinary guidance. Lancet Respir Med. 2020;8:717-25.

29. Guidelines for disinfection and sterilization in healthcare facilities 2008. Centers for disease control and prevention. Available at: https://www.cdc.gov/ infectioncontrol/guidelines/disinfection/recommenda tions.html. Accessed on 10 April 2020.

30. McGrath BA, Bates L, Atkinson D, Moore JA; National Tracheostomy Safety Project. Multidisciplinary guidelines for the management of tracheostomy and laryngectomy airway emergencies. Anaesthesia. 2012;67(9):1025-41.

31. Chan JYK, Wong EWY, Lam W. Practical Aspects of Otolaryngologic Clinical Services During the 2019 Novel Coronavirus Epidemic: An Experience in Hong Kong. JAMA Otolaryngol Head Neck Surg. 2020;146(6):519-20. 
32. Zuo MZ, Huang YG, Ma WH. Expert Recommendations for Tracheal Intubation in Critically ill Patients with Noval Coronavirus Disease 2019. Chin Med Sci J. 2020;35(2):105-109.

33. Ten Hoorn S, Elbers PW, Girbes AR, Tuinman PR. Communicating with conscious and mechanically ventilated critically ill patients: a systematic review. Crit Care. 2016;20(1):333.

34. Rovira A, Dawson D, Walker A, et al. Tracheostomy care and decannulation during the COVID-19 pandemic. A multidisciplinary clinical practice guideline. Eur Arch Otorhinolaryngol. 2020;1-9.

35. Tornari C, Surda P, Takhar A, et al. Tracheostomy, ventilatory wean, and decannulation in COVID-19 patients. Eur Arch Otorhinolaryngol. 2020;1-10.

36. Mishra P, Jedge P, Kaushik M, Artham P, Kumari S. Our Experience of Tracheostomy in COVID-19 Patients . Indian J Otolaryngol Head Neck Surg. 2020;1-4.

37. Chao TN, Harbison SP, Braslow BM, et al. Outcomes after Tracheostomy in COVID-19 Patients. Ann Surg. 2020;272(3):e181-6.

38. Angel L, Kon ZN, Chang SH, et al. Novel Percutaneous Tracheostomy for Critically Ill Patients
With COVID-19. Ann Thorac Surg. 2020;110(3): 1006-11.

39. Wang Y, Lu X, Li Y, Chen H, Chen T, Su N, Huang F, Zhou J, Zhang B, Yan F, Wang J. Clinical Course and Outcomes of 344 Intensive Care Patients with COVID-19. Am J Respir Crit Care Med. 2020 Jun 1;201(11):1430-4.

40. Zhang J, Wang X, Jia X, Li J, Hu K, Chen G, et al. Risk factors for disease severity, unimprovement, and mortality in COVID-19 patients in Wuhan, China. Clin Microbiol Infect. 2020;26(6):767-72.

41. Garg S, Kim L, Whitaker M, O’Halloran A, Cummings C. Hospitalization rates and characteristics of patients hospitalized with laboratory-confirmed coronavirus disease 2019COVID-NET, 14 states. Morb Mortal Wkly Rep. 2020;69(15):458-64.

Cite this article as: Juvekar M, Sarkar B. Guidelines for tracheostomy in COVID-19 pandemic. Int J Otorhinolaryngol Head Neck Surg 2020;6:2328-33. 